\title{
Reply
}

Neuropsychobiology

\section{SyNoPsis: Response to the Commentators}

\author{
Werner Strik Katharina Stegmayer Sebastian Walther Thomas Dierks \\ University Hospital of Psychiatry, University of Bern, Bern, Switzerland
}

\section{Keywords}

SyNoPsis · Schizophrenia · Psychosis · Bern

Psychopathology Scale $\cdot$ Systems neuroscience

In their commentary, Manuel Cuesta and Víctor Peralta [1] acknowledge several of the basic contributions and challenges of our SyNoPsis project. They appreciate the attempt to link clinical manifestations with the neurobiology of schizophrenia by means of a revised semiology, and the proposal to understand its essence as a disorder of interpersonal communication. The authors also recognize the aspects contributed by the important work of Carl Wernicke and Karl Leonhard [2, 3].

Also, they carve out some relevant limitations of SyNoPsis. Although a relationship of specific symptom dimensions to the respective sensorimotor and corticostriatal brain systems has been shown in several studies [4], the functional specificity of these systems has yet to be defined [5]. Further, different within-systems dynamics may result in different symptom patterns, and the anatomical connections and functional interactions between the systems have not yet been sufficiently understood.

In their commentary, Cuesta and Peralta raise two critical clinical aspects: the course of the illness, and the representation of negative symptoms. We agree that the course of psychotic disorders is of eminent importance for clinical management, but also for understanding the long-term dynamics of the brain systems involved. In fact, our clinical instrument, the Bern Psychopathology Scale (BPS) [6], was designed as an instrument for crosssectional assessment. However, it allows recurrent assessments at deliberate intervals for follow-up observations. First retrospective studies have recently been published [7], and the issue of the course of psychosis dimensions will be addressed in detail in future projects.

Regarding negative symptoms, we pursued a novel approach, mapping "negative" behavior onto inhibitory states of the respective brain functions. Following our objective to revise the semiology of psychosis based on the best of our knowledge about the related brain functions, this group of symptoms was disentangled respecting the boundaries of higher brain functions. For instance, we conceptualize alogia as "inhibition of verbal expression," and avolition as "lack of spontaneous actions." Thus, negative symptoms are redefined according to the tripartite symptom structure of SyNoPsis to match dysfunctions of the three brain circuits in question. In fact, most overlap was found between the motor dimension and negative symptoms [8].

\section{KARGER}

(c) 2017 S. Karger AG, Basel 
Vijay Mittal [9] picks up the similarities and differences between SyNoPsis and RDoC, highlighting the revised semiology of our project in order to allow a testable, direct link of symptoms with brain systems, without the need of intermediate constructs. Further, he acknowledges the dimensional approach in terms of positive and negative deviations from normal experience and behavior, the focus on interpersonal communication, and, in the light of his own contributions [10] regarding the motor circuitry, the awareness of the contribution of the frontosubcortical circuits to complex behavior.

As future challenges for SyNoPsis, he delineates the prospect of extending the focus on shared and distinct phenomenology across mental disorders, on comorbidity, and on the underlying biological building blocks and modulators of the systems at molecular, cellular, and physiological levels. Further, he highlights the explicitly elaborated theoretical background as both a strength and a risk, emphasizing that it is important for SyNoPsis to continue to develop and adapt according to novel findings and alternative viewpoints. We fully agree with this claim. SyNoPsis is not designed as a dogmatic doctrine, but as an adaptive framework for empirical research.

Introducing his commentary on SyNoPsis, Peter Falkai [11] recalls Wilhelm Griesinger's statement that mental disorders are brain disorders, with the consequent necessity to simplify hypotheses and to map psychopathological features onto brain structures and brain functions. He remarks the decades of our group's work regarding the SyNoPsis project, as well as the thorough revision of the clinical vocabulary, which introduces three neurobiologically informed behavioral dimensions. Dr. Falkai acknowledges the potential of the novel semiotics to develop individual treatment strategies and therapeutic alliance, and the valuable framework for didactic purposes provided by SyNoPsis.

As a further development, he suggests complementing SyNoPsis with hypothesis-free analyses for potentially fruitful comparisons. Finally, he correctly remarks that possible genetic and nongenetic risk factors as significant parts of the pathophysiology of schizophrenia are still left out of the project.

In his thoughtful and historically well-informed commentary, Stephan Heckers first summarizes good reasons why schizophrenia research needs conceptual innovation to escape a dead end. He highlights analogies between the approach chosen by SyNoPsis and well-established models in neurology, and states that there is no a priori reason that this approach should not work for psychosis.
He raises, however, questions about the project. Similar tripartite symptom structures of psychosis have been proposed by illustrious 19th- and early-20th-century psychiatrists, and there is still need for conceptual clarification - for instance, regarding the scientific evidence for the "natural segregation" of the three domains and their mutual relationships, and whether the SyNoPsis behavioral dimensions are intended as an exhaustive list.

We are aware of these issues and emphasize that our symptom dimensions are neither intended nor able to cover all facets of human behavior and experience. Instead, they focus on core functions of the human brain crucial for interpersonal communication. The related systems are understood as parallel modules for specialized brain functions, which at the highest level of brain organization are integrated into a unique consciousness and complex actions. A "dissociation" of these systems may account for function-specific deficits, but it will not explain all integrated functions or compensatory reactions of the orchestrated brain.

This is partially related to the question of how we understand the relationship between the domains. Based on our psychopathological studies and our knowledge about functional anatomy, a dimensional model better applies to the domains than categories. In other words, we do not assume orthogonal, but parallel, interacting relationships. Furthermore, in an ongoing research project, we are investigating the question of natural segregation of the related psychic capacities in healthy persons.

Another issue raised by the commentator addresses the functional boundaries of the symptom-systems maps. This is a very intriguing and legitimate question. We have, in fact, found activation of the inferior frontal gyrus (Broca's region) during meaningful hand gestures [12], suggesting a contribution of the language network to any semantic expression. On the other hand, we have found clear covariations between disorders of the motor and the language domain in the BPS $[6,13]$. It will be hard scientific work of multiple disciplines to understand whether these and other functional overlaps are based on low-level, anatomically "hard-wired" shortcuts between the systems at the basal ganglia or cortical level, or the result of cumbersome learning like linking our language capacities to visual object recognition (reading) and fine motor behavior (writing).

In perfect accordance with our own perception, Dr. Heckers states that the ultimate test of the SyNoPsis project will be the prediction of treatment responses and illness trajectories using systems neuroscience models [14]. 
William Carpenter outlines the state of schizophrenia research in the light of the past few decades of neurobiological research, showing that progress towards a neurobiologically informed psychopathology has been much slower than expected [15]. This is manifest in the lack of knowledge for the DSM-5 regarding brain mechanisms. $\mathrm{He}$ then underlines the need for reducing heterogeneity in the clinical syndrome of schizophrenia. His personal view expressed in 1974 [16] for several aspects anticipates some pivotal SyNoPsis concepts, such as a tripartite model with six symptom domains, positive and negative poles of the symptomatology, and a focus on the interpersonal context and on the clinical heterogeneity which severely compromises hypothesis falsification. Further, he acknowledges the ambition of SyNoPsis to target the gap between brain systems and clinical symptoms, without the need of intermediate constructs.

We very much appreciate Dr. Carpenter's recognition of two aspects, which from the beginning have been crucial personal motivations for our project: a thorough and respectful but at the same time conceptually consistent and scientifically valid psychopathology, and a continuous, close exchange with clinical care.

All commentators in one way or the other acknowledge the direct link between brain functions and the symptom level of psychosis, and at the same time stress that first results and observations in this direction must find their way into clinical care to prove the validity of the project. Among the next steps suggested on this route are translations between the different current clinical and diagnostic concepts of schizophrenia, and an explicit demarcation between BPS symptoms and all the psychic and behavioral phenomena which are not covered by SyNoPsis. This is, of course an endeavor which cannot be accomplished by a single research group. The results of our current collaborations suggest that the SyNoPsis concept can produce similar results in other laboratories. Thus, multisite studies on SyNoPsis are possible. Finally, we hope that this special issue of Neuropsychobiology will stimulate and inspire debate on the necessary "course corrections for the schizophrenia field caught in rough waters" [15].

\section{References}

1 Cuesta MJ, Peralta V: A new step forward in "carving nature by its joints" in psychopathology/psychosis. Neuropsychobiology 2017; 75:117-118

2 Wernicke C: Grundriss der Psychiatrie in klinischen Vorlesungen. Leipzig, Verlag von Georg Thieme, 1900, vol 1, p 201.

3 Leonhard K: Aufteilung der endogenen Psychosen und ihre differenzierte Ätiologie, ed 8 . Stuttgart/New York, Thieme, 2003.

4 Strik W, Stegmayer K, Walther S, Dierks T: Systems Neuroscience of Psychosis: mapping schizophrenia symptoms onto brain systems. Neuropsychobiology 2017;75:100-116.

5 Spunt RP, Adolphs R: A new look at domain specificity: insights from social neuroscience. Nat Rev Neurosci 2017;18:559-567.

6 Strik W, Wopfner A, Horn H, Koschorke P, Razavi N, Walther S, Wirtz G: The Bern Psychopathology Scale for the assessment of system-specific psychotic symptoms. Neuropsychobiology 2010;61:197-209.
7 Wigand ME, Lang FU, Becker T, Schulze TG, Strik W, Jäger M: Psychopathological course typology in schizophrenia spectrum disorders: a heuristic approach in a sample of 100 patients. Psychopathology 2016;49:397-405.

8 Lang FU, Walther S, Stegmayer K, AndersonSchmidt H, Schulze TG, Becker T, Jäger M: Subtyping schizophrenia: a comparison of positive/negative and system-specific approaches. Compr Psychiatry 2015;61:115121.

9 Mittal VA: Systems Neuroscience of Psychosis (SyNoPsis) provides a promising framework for advancing the field. Neuropsychobiology 2017;75:119-121.

10 Mittal VA, Bernard JA, Northoff G: What can different motor circuits tell us about psychosis? An RDoC perspective. Schizophr Bull 2017;43:949-955.

11 Falkai P: Commentary on "Systems Neuroscience of Psychosis (SyNoPsis): mapping schizophrenia symptoms onto brain systems." Neuropsychobiology 2017;75:122-123.
12 Stegmayer K, Bohlhalter S, Vanbellingen T, Federspiel A, Wiest R, Müri RM, Strik W, Walther S: Limbic interference during social action planning in schizophrenia. Schizophr Bull 2017, Epub ahead of print.

13 Lang FU, Stierlin AS, Stegmayer K, Walther S, Becker T, Jäger M: Factor structure of the Bern Psychopathology Scale in a sample of patients with schizophrenia spectrum disorders. Eur Psychiatry 2015;30:880-884.

14 Heckers S: SyNoPsis: disruptive innovation for mapping schizophrenia onto the brain. Neuropsychobiology 2017;75:124-125.

15 Carpenter WT: Deconstructing schizophrenia. Neuropsychobiology 2017;75:126-128.

16 Strauss JS, Carpenter WT Jr, Bartko JJ: The diagnosis and understanding of schizophrenia. Part III. Speculations on the processes that underlie schizophrenic symptoms and signs. Schizophr Bull 1974;11:61-69. 